\title{
The effect of myocardial anisotropy on volume measurement using conductance catheter techniques in the right ventricle
}

\author{
Chanchai Thaijiam ${ }^{1} \quad$ Timothy John Gale ${ }^{2}$
}

(Received 27 July 2007; revised 2 October 2008)

\begin{abstract}
The effect of myocardial anisotropy on ventricular volume measurements, taken using conductance catheter techniques in the right ventricle, is investigated using finite element models of the isolated heart. The governing equation is Poisson's equation. Model results show calculated volumes are more accurate if anisotropy is included in the model, due to increased confinement of current within the ventricle. This can lead to more accurate blood volume measurements in the right ventricle.
\end{abstract}

\section{Contents}

1 Introduction

See http://anziamj.austms.org.au/ojs/index.php/ANZIAMJ/article/view/331 for this article, (c) Austral. Mathematical Soc. 2008. Published October 7, 2008. ISSN 1446-8735 
2 Methodology

2.1 Conductance technique theory . . . . . . . . . . . C735

2.2 Finite element modelling . . . . . . . . . . . . . . . C736

2.2.1 Finite element principle . . . . . . . . . . C737

2.2.2 Model geometry . . . . . . . . . . . . . C738

2.3 Investigations of parallel conductance . . . . . . . . . C741

3 Results

4 Discussion

C742

5 Conclusion

C746

References

C746

\section{Introduction}

Conductance catheterization is only one technique for continuous measurement of intraventricular blood volume in an implanted system. In its simplest form, A.C. electric current is passed between distal electrodes on a catheter, the resulting time-varying voltage is measured at intermediate locations, and the electric field is assumed to be contained within the ventricular chamber with a uniform distribution parallel to the major axis [1]. Intraventricular blood volume is calculated using basic equations of electric field theory [8], involving calculating the segmental resistance between adjacent measurement electrodes knowing the potential drop across each volume segment.

However, there are obstacles to achieving accurate volume measurement using this method, especially with the complex shape of the right ventricle, which is the preferred location for an implanted system [12,3,11]. A primary source of error is electric current leakage (parallel conductance) through surrounding tissues, which have a finite resistance, leading to overestimation of 
conductance volume [2]. Also, a complicating factor is that the heart tissue (myocardium) is anisotropic, with higher electrical conductivity along the myocardial fibers than transverse to the fibers. We are interested in using a numerical model to establish the effect of parallel conductance and in particular the influence of myocardial anisotropy on the accuracy of volume measurement. This may reveal how parallel conductance and myocardial anisotropy alter the distribution of conductance current within the heart, knowledge that may potentially be used to develop improved techniques. Numerical modelling methods have previously been used to estimate parallel conductance but have invariably assumed isotropic conditions, ignoring anisotropic conductance from myocardial fiber orientation. Here we use Finite Element models to explore the effect of myocardial anisotropy on parallel conductance measurements using conductance catheter techniques in the isolated heart. A simple block model and a realistic isolated heart model (with and without anisotropy) are presented in Section 2.2.2.

\section{Methodology}

\subsection{Conductance technique theory}

Figure 1 shows a model of a conductance catheter in a ventricle. Constant electric current is injected through the source and sink electrodes, and the segmental resistance is calculated knowing the potential drop across each volume segment [1],

$$
R_{i}=\frac{\rho_{b} L_{i}}{A_{i}}=\frac{\rho_{b} L_{i}^{2}}{A_{i} L_{i}}=\frac{\rho_{b} L_{i}^{2}}{\nu_{i}}
$$

where $R_{i}=$ segmental resistance, $\rho_{b}=$ blood resistivity, $L_{i}=$ distance between any two measurement electrodes, $A_{i}=$ cross sectional area of the segment, and $v_{i}=$ segmental volume. 


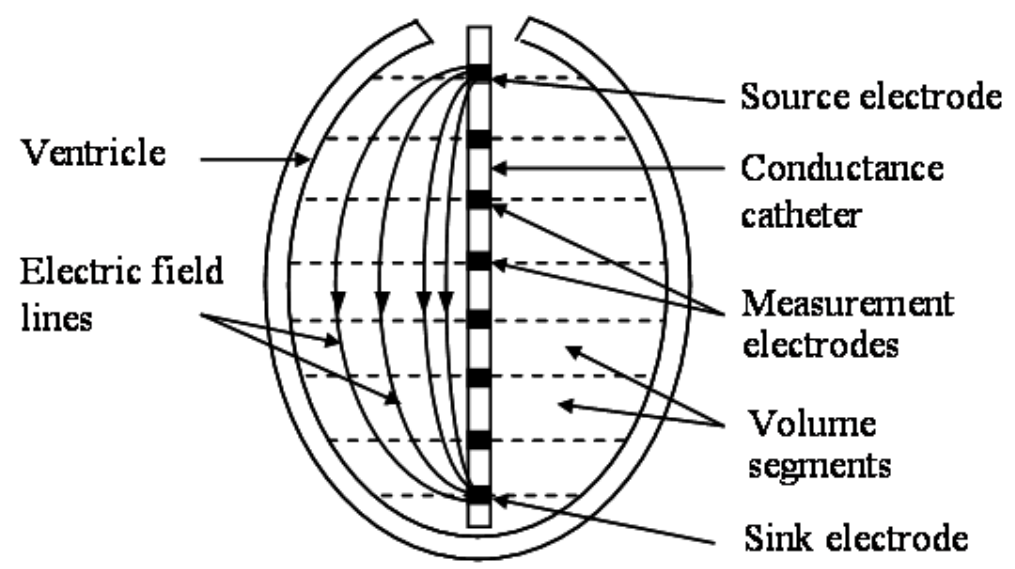

FIGURE 1: Model of a conductance catheter inserted into a ventricle.

The segmental volume is found from (1) as

$$
v_{i}=\frac{\rho_{\mathrm{b}} \mathrm{L}_{\mathrm{i}}^{2}}{\mathrm{R}_{\mathrm{i}}} .
$$

Knowing the segment volumes, we find the total ventricular volume as

$$
v_{\text {total }}=\sum_{i=1}^{p} v_{i}
$$

where $p=$ total number of volume segments.

\section{$2.2 \quad$ Finite element modelling}

The electric potential distribution inside the finite element model is found using Poisson's equation [6, 4]:

$$
\nabla^{2} \mathrm{~V}=-\frac{\mathrm{I}}{\sigma}
$$


where $\mathrm{V}=$ electric potential, $\sigma=$ material conductivity, and $\mathrm{I}=$ current density.

The continuity conditions between adjacent elements (materials 1 and 2) are

$$
\begin{aligned}
\mathrm{V}_{1} & =\mathrm{V}_{2}, \\
\sigma_{1} \nabla \mathrm{V}_{1} \cdot \overrightarrow{\mathrm{a}}_{\mathrm{N}} & =\sigma_{2} \nabla \mathrm{V}_{2} \cdot \overrightarrow{\mathrm{a}}_{\mathrm{N}} .
\end{aligned}
$$

Equation (5) represents continuous electric potential across the boundary. Equation (6) represents the continuity of electric current density in a direction $\vec{a}_{N}$ normal to the boundary.

\subsubsection{Finite element principle}

Poisson's equation (4) is solved by the finite element method by finding a potential function $\mathrm{V}$ that minimized the energy related functional,

$$
\mathrm{F}(\mathrm{V})=\int\left\{\frac{1}{2}\left[\sigma|\nabla \mathrm{V}|^{2}\right]-\mathrm{IV}\right\} \mathrm{d} v
$$

The region of integration is the entire volume of the model, subdivided into elements. The potential within each element, $V_{e}$, is represented by a linear combination of interpolating polynomials:

$$
\begin{aligned}
\mathrm{V}_{e}(x, y, z) & =\sum_{m} \mathrm{~N}_{m}(x, y, z) \mathrm{V}_{\mathrm{m}} \\
\nabla \mathrm{V}_{e}(x, y, z) & =\sum_{m} \nabla \mathrm{N}_{\mathrm{m}}(x, y, z) \mathrm{V}_{\mathrm{m}}
\end{aligned}
$$

where $N_{m}$ are shape functions and $V_{m}$ are the potentials at local nodes $m$ of the element. To minimize the energy related functional $[6,4]$, we set 
$\partial F(V) / \partial\left(V_{n}\right)=0$, where $n$ is all nodes (global nodes) of the entire model. Finally, we rewrite (7) in matrix form:

$$
[\mathrm{K}] \mathrm{V}=\mathrm{f}
$$

where $[\mathrm{K}]$ is a stiffness matrix or a global coefficient matrix, $\mathrm{V}$ is a column vector of global nodal potentials, and $f$ is a column vector of loads (current loads, for example). As $[K]$ and $f$ are known, we find $V$ at nodes of the entire model.

\subsubsection{Model geometry}

Two finite element model geometries were constructed: a simple block model for investigation of basic principles; and a heart model for investigation in realistic geometry. Models were constructed using ANSYS 10 multi-physics software (ANSYS, Inc., Canonsburg, USA).

Simple block model geometry The simple block model consisted of two concentric cuboidal conductors with different material properties, Figure 2. The inner conductor was an analogy to the right ventricular chamber, while the outer conductor represented myocardium. Anisotropic fiber orientation was parallel to the outer wall. Dimensions of the inner and outer conductors were $-35 \leq x \leq 35,-35 \leq y \leq 50$, and $-110 \leq z \leq 110 \mathrm{~mm}$, and $-50 \leq x \leq 50,-50 \leq y \leq 50$, and $-125 \leq z \leq 125 \mathrm{~mm}$, respectively. Current delivery electrodes (source and sink) were spaced at $\mathrm{L}=140 \mathrm{~mm}$ and located asymmetrically (offset along the $y$-axis), representing positions used in the right ventricle $[8,10,9]$. Six measurement electrodes (five segments) were located at $x=y=0$ and parallel to the $z$-axis at $20 \mathrm{~mm}$ intervals. Distal measurement electrodes were at $z= \pm 50 \mathrm{~mm}$. The model was discretised into 150,000 tetrahedral finite elements. The sink and source current were the same at $x=a=0, y=b=30$ but different at $z=-\mathrm{L} / 2$, and $\mathrm{L} / 2$, respectively. 


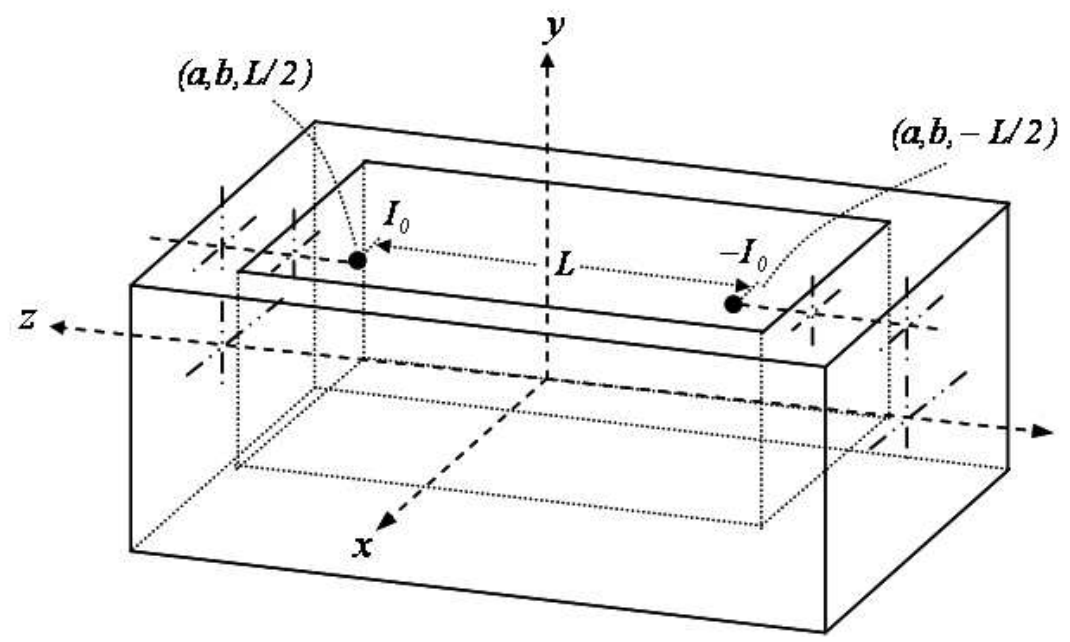

Figure 2: Block model geometry.

Heart model geometry The heart model, Figures 3 and 4, was constructed from the "Auckland model" data [5] defining geometric and material properties in 3D for the canine heart. Each anisotropic myocardium element was assigned different conductivities in three directions corresponding to the myocardial fiber vectors. This was achieved by Euler rotation angles. A curved conductance catheter was introduced into the right ventricle, Figure 5, as done by Gopakumaran et al. [3]. The model was discretised into 150,000 tetrahedral finite elements.

Material property The myocardium was modeled as either isotropic, with conductivity of $0.135 \mathrm{~S} / \mathrm{m}$, or anisotropic, with longitudinal and transverse conductivities of $0.21 \mathrm{~S} / \mathrm{m}$ and $0.06 \mathrm{~S} / \mathrm{m}$, respectively [7]. Blood conductivity was $0.67 \mathrm{~S} / \mathrm{m}[2]$. 


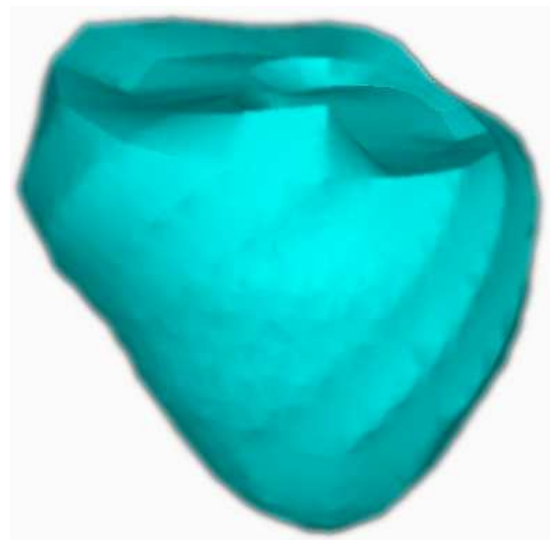

Figure 3: Finite element canine model of the heart.

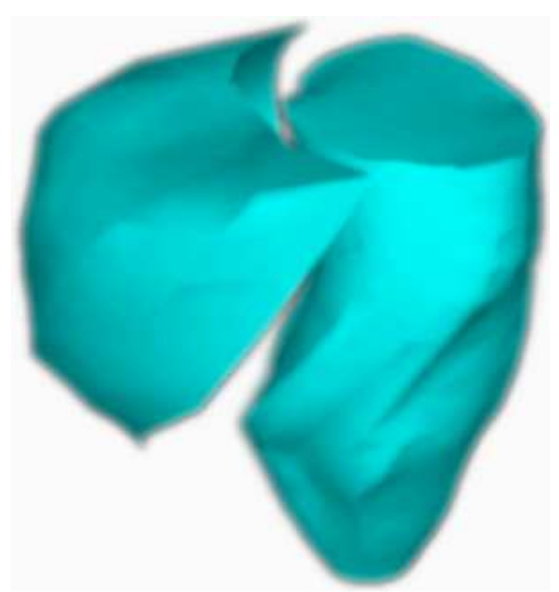

FiguRE 4: Finite element canine model of the right and left ventricles. 


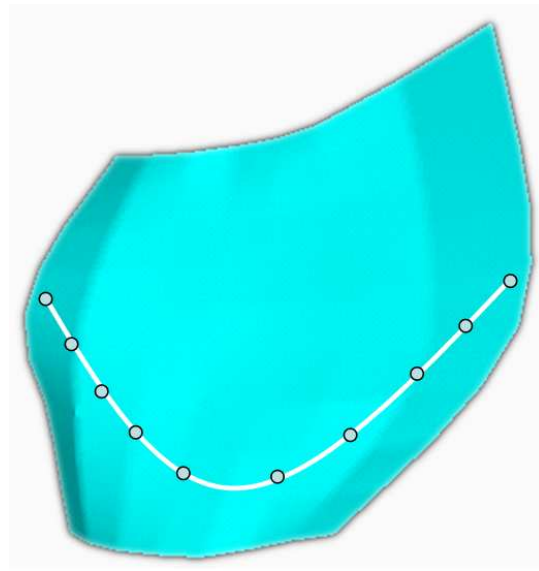

FiguRE 5: Catheter position within the canine right ventricular chamber.

Application of current source and sink Boundary conditions consisted of applied currents of $20 \mu \mathrm{A}$ on two specified nodes representing source and sink electrodes. The current sink was also constrained by a voltage of zero.

Finite element solution A numerical solution using the finite element method was employed, using ANSYS (running on a $2.80 \mathrm{GHz}$ PC under Windows XP).

\subsection{Investigations of parallel conductance}

Investigations were done using the specified conductance catheter configuration for each of the block and heart models. The block model was configured first as an isolated ventricle (inner conductor alone) and then as inner conductor surrounded by isotropic myocardium, and inner conductor surrounded by anisotropic myocardium. Similarly, the canine model was configured first as an isolated right ventricular chamber, then as a complete 
heart model consisting of left and right ventricles and myocardium (isotropic and anisotropic). True volumes were calculated between distal measurement electrodes, and conductance volumes, Section 2.1, were calculated for each configuration. The difference in the conductance volumes was the true leakage volume (parallel conductance). The maximum and average ventricular flux densities were also calculated for each configuration.

\section{$3 \quad$ Results}

Numerical results were calculated for conductance volume and leakage volume (parallel conductance) for the block model, Table 1, and for the canine model, Table 2. The total true volume within the block ventricle and canine ventricle was 595 and $27.86 \mathrm{~cm}^{3}$, respectively. Values were calculated for nodal electric flux density within the inner block and right ventricular chamber, Tables 3 and 4, respectively.

\section{Discussion}

Calculated conductance volumes for both the isolated block ventricle and the isolated canine ventricle were relatively accurate, Tables 1 and 2. However, the errors for the isolated ventricles were produced as the assumption that the electric field distribution within a model is a uniform field [10]. Furthermore, the conductance catheter in the canine model did not span the whole ventricular chamber, and this resulted in a higher error than for the block model. When parallel conductance was added, there was significantly more error in calculated ventricular volumes, due to the addition of parallel pathways, Tables 1 and 2. There was greater error, and larger parallel volume, for isotropic than anisotropic myocardium. The improvement for the anisotropic case may be explained by considering that myocardial electric 
TABLE 1: Conductance and parallel conductance volumes $\left(\mathrm{cm}^{3}\right)$ for the block model.

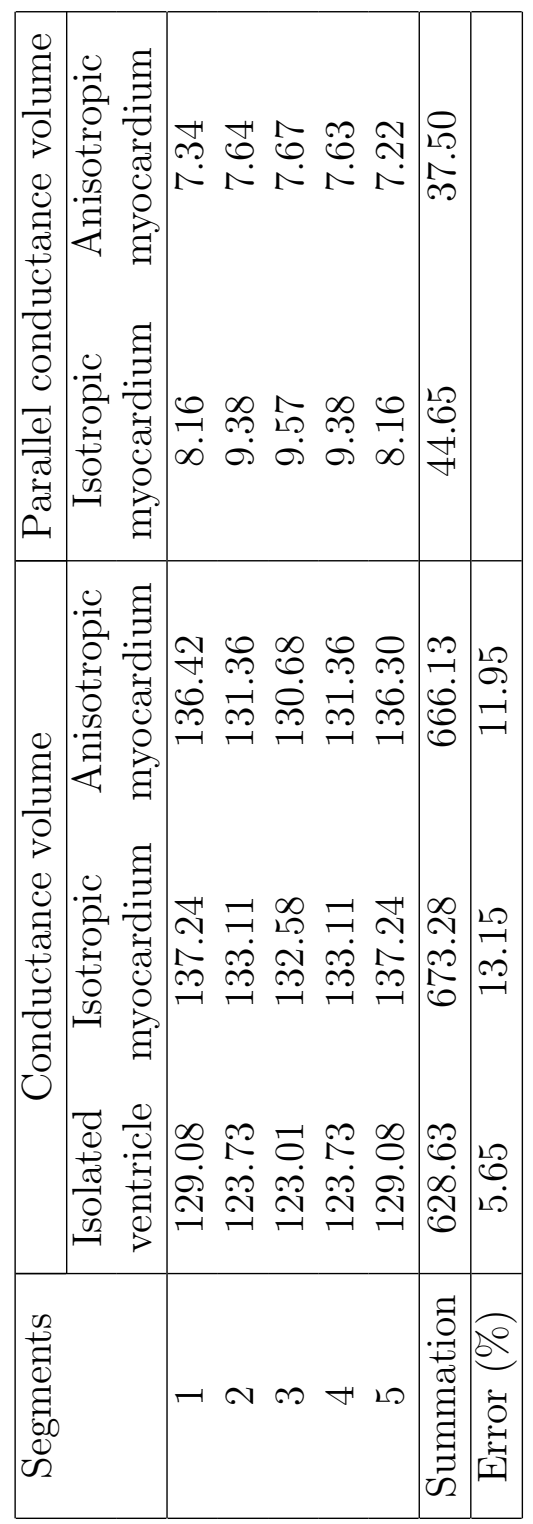


TABLE 2: Conductance and parallel conductance volumes $\left(\mathrm{cm}^{3}\right)$ for the heart model.

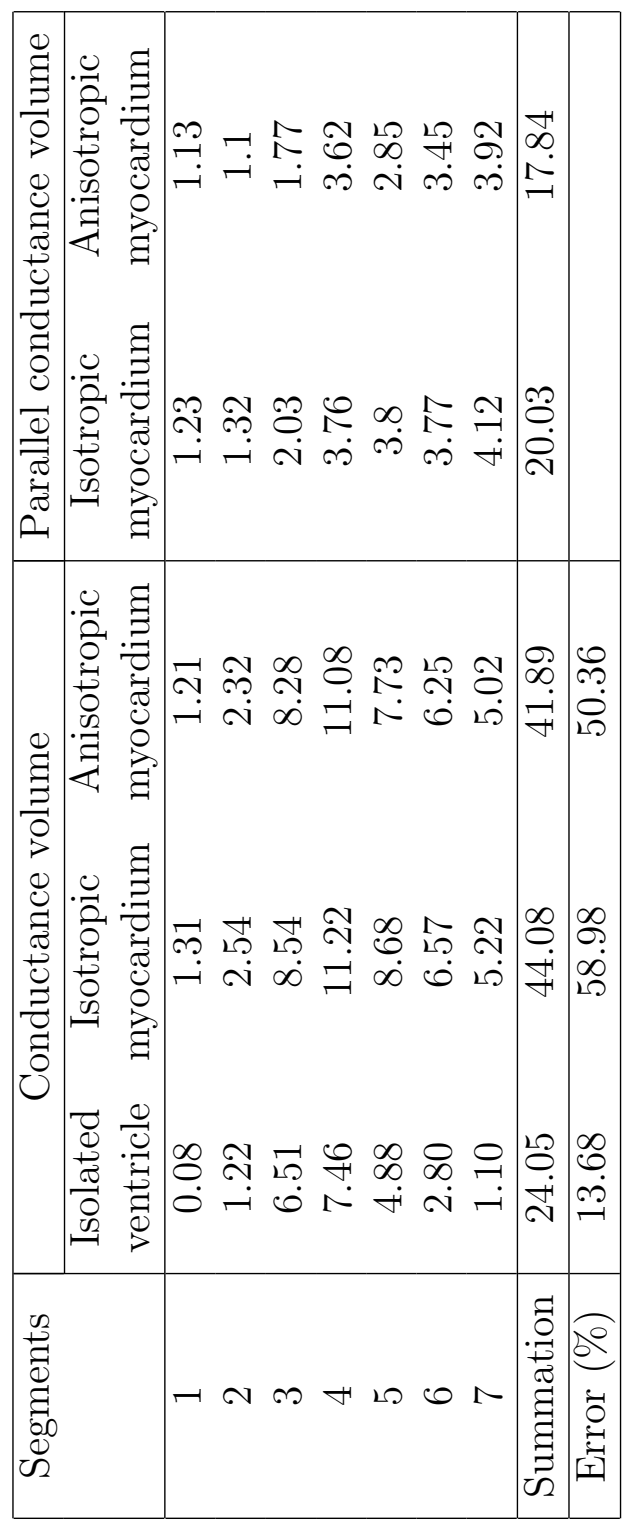


TABLE 3: Electric flux densities $\left(\mathrm{C} / \mathrm{m}^{2}\right)$ within the right ventricle for the block model.

\begin{tabular}{|c|ccc|}
\hline Values & $\begin{array}{c}\text { Isolated } \\
\text { Ventricle }\end{array}$ & $\begin{array}{c}\text { Isotropic } \\
\text { Myocardium }\end{array}$ & $\begin{array}{c}\text { Anisotropic } \\
\text { Myocardium }\end{array}$ \\
\hline Maximum & 0.0094 & 0.0054 & 0.0065 \\
Average & 0.0029 & 0.0020 & 0.0022 \\
\hline
\end{tabular}

TABLE 4: Electric flux densities $\left(\mathrm{C} / \mathrm{m}^{2}\right)$ within the right ventricle for the heart model.

\begin{tabular}{|c|ccc|}
\hline Values & $\begin{array}{c}\text { Isolated } \\
\text { Ventricle }\end{array}$ & $\begin{array}{c}\text { Isotropic } \\
\text { Myocardium }\end{array}$ & $\begin{array}{c}\text { Anisotropic } \\
\text { Myocardium }\end{array}$ \\
\hline Maximum & 2.4334 & 0.0680 & 0.0702 \\
Average & 0.2546 & 0.0142 & 0.0146 \\
\hline
\end{tabular}


resistivity was lower in the longitudinal direction than in the transverse direction. Therefore, the electric field distribution was more confined within the ventricular cavity for the anisotropic case as the leakage current preferentially flowed along fiber directions. This limited current flow more to the ventricular chamber. This was confirmed by the observation of higher electric flux density within the ventricle for anisotropic than for isotropic myocardium, Tables 3 and 4 . The conclusion is that leakage current can penetrate the myocardial wall less easily in the anisotropic case than in the isotropic case.

\section{Conclusion}

In summary, the electric potential distribution within the ventricle changed according to the surrounding material properties. The electric potential distribution was confined more within the inner regions of the models when the surrounding material was anisotropic, resulting in higher electric flux density within the ventricle. This resulted in a small improvement in accuracy of the conductance catheterization technique.

Acknowledgements The authors gratefully acknowledge the contribution of Prof. Dr. Peter J. Hunter, The University of Auckland, New Zealand, for making available canine heart geometry data.

\section{References}

[1] J. Baan, T. T. Aouw Jong, P. L. M. Kerkhof, R. J. Moene, A. D. Van Dijk, E. T. Van Der Velde, and J. Koops. Continuous stroke volume and cardiac output from intra-ventricular dimensions obtained with 
impedance catheter. Cardiovascular Research, 15:328-334, 1981. C734, C735

[2] L. A. Geddes and L. E. Baker. The specific resistance of biological meterial: a compendium of data for the biomedical engineer and physiologist. Med. Biol. Eng., 5:271-293, 1967. C735, C739

[3] B. Gopakumaran, J. H. Petre, S. Krucinski, and P. Murray. Real-time continuous measurement of right ventricular volume using a conductance catheter. Biom. Instrum. \& Techno., 30(5):427-438, 1996. C734, C739

[4] C. Johnson. Numerical solution of partial differential equations by the finite element method. Cambridge University Press, 1990. C736, C737

[5] P. M. Nielsen, I. J. Le Grice, B. H. Smaill, and P. J. Hunter. Mathematical model of geometry and fibrous structure of the heart. American Journal of Heart and Circulatory Physiology, 260:H1365-H1378, 1991. C739

[6] P. P. Silvester and R. L. Ferrari. Finite elements for electrical engineer. Cambridge University Press, 1996. C736, C737

[7] J. G. Stinstra, B. Hopenfeld, and R. S. Macleod. On the passive cardiac conductivity. Ann. Biomed. Eng., 33:1743-1751, 2005. C739

[8] C. Thaijiam and T. J. Gale. Analytical and numerical studies of volume measurement using conductance catheter techniques. In Proceedings of the 2005 IEEE Engineering in Medicine and Biology Society 27th Annual Conference, pages 2291-2294, 2005. C734, C738

[9] C. Thaijiam and T. J. Gale. The effect studies of fiber orientation with volume measurement by using conductance catheter techniques. In Proceedings of the 2006 IEEE Engineering in Medicine and Biology Society 28th Annual Conference, pages 5981-5984, 2006. C738 
[10] C. Thaijiam and T. J. Gale. Studies of single and dual excitation using conductance catheter techniques. In The Fourth IASTED

International Conference on Biomedical Engineering, pages 294-299, 2006. C738, C742

[11] P. A. White and A. N. Redington. Right ventricular volume measurement: can conductance do it better? Physio. Meas., 21:R23-R41, 2000. C734

[12] J. C. Woodard, C. D. Bertram, and B. S. Gow. Dectecting right ventricular volume changes using the conductance catheter. PACE, 15, 1992. C734

\section{Author addresses}

1. Chanchai Thaijiam, School of Engineering, University of Tasmania, Hobart, Australia

mailto:ct0@postoffice.utas.edu.au

2. Timothy John Gale, School of Engineering, University of Tasmania, Hobart, Australia 